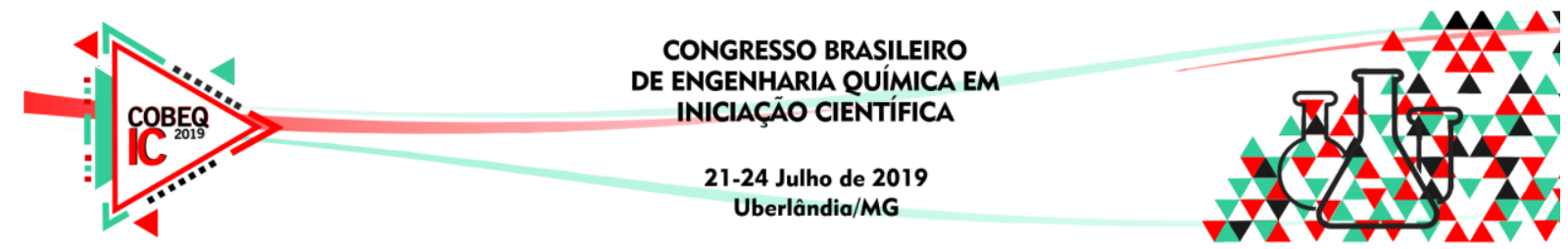

\title{
AVALIAÇÃO DO USO DE CASCA DE COCO VERDE PARA A PRODUÇÃ̃ DE CARVÃO VEGETAL
}

\author{
T. H. G. SILVA ${ }^{1}$, G. R. STROHER ${ }^{1}$, G. L. STROHER ${ }^{1}$ \\ ${ }^{1}$ Universidade Tecnológica Federal do Paraná \\ E-mail para contato: giselys@utfpr.edu.br
}

\begin{abstract}
RESUMO - Com a finalidade de promover a destinação adequada para a casca de coco verde, o presente trabalho mostra uma solução viável com a transformação dos resíduos do coco em carvão ativado. Foram realizados experimentos comparativos do carvão produzido neste trabalho com carvão ativado disponível no mercado, os resultados mostraram superioridade do carvão deste trabalho em relação ao carvão ativado comercial. Do mesmo modo foram realizados testes para aferir a eficiência do carvão ativado produzido, avaliando-se qual massa de carvão apresentaria melhores resultados de adsorção. Nas melhores condições de ativação por cloreto de zinco II os resultados mostram que apenas $0,109 \%$ do rejeito de lavanderia analisado não foram adsorvidos denotando alta eficiência quando comparado com carvão encontrado no mercado.
\end{abstract}

\section{INTRODUÇÃO}

O Brasil produz anualmente cerca de 1 bilhão de cocos verdes e secos, onde apenas $10 \%$ é reciclado (BBC BRASIL, 2014). Em dezembro de 2013 chegaram em São Paulo cerca de 1,7 milhões de unidades de coco onde apenas sua água foi aproveitada, gerando em média $1,75 \mathrm{~kg} /$ unidade de resíduo. Sem iniciativas para reciclagem todo material residual gerado acaba indo para aterros sanitários ou descartados de maneira irregular. (CARRIJO et al., 2002).

O descarte irregular da casca de coco acarreta alguns problemas de saúde pública como a proliferação de doenças, dentre elas: dengue, febre amarela, Chikungunya, zika, o mosquito transmissor destas doenças utiliza a água parada na casca do coco para se proliferar. (PALMA, 2014).

\subsection{Caracterização da Casca de Coco Verde}

A fruta apresenta uma estrutura fibrosa, mas com uma superfície lisa (epicarpo). As cores do fruto podem variar de verde a marrom avermelhado. O feixe de fibras (mesocarpo) é 


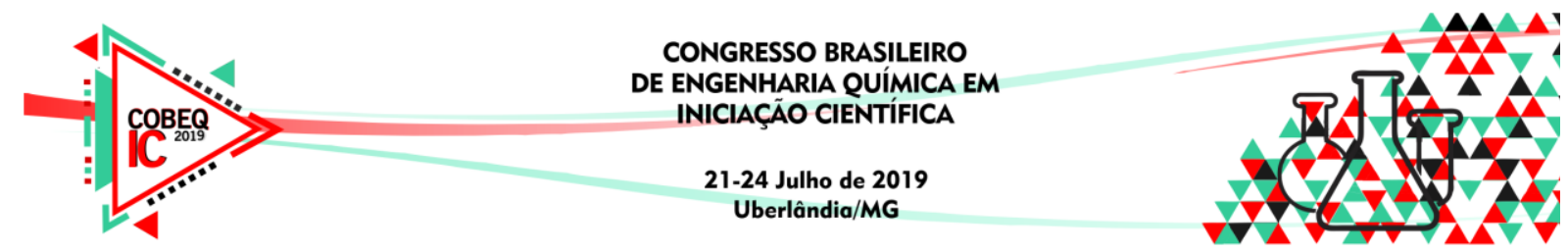

branco e composto majoritariamente por lignina e glucose. Mais adentro se encontra uma casca dura (endocarpo) que envolve o núcleo (endosperma) (BAUDOUIN et al., 1996).

\subsection{Carvão de Coco}

A casca do coco é um material orgânico composto principalmente por lignina glucose e possui uma estrutura similar com a madeira que é utilizada na produção de carvão vegetal. (ANDRADE, 2004). Segundo Nogueira (2000) o coco pode ser queimado diretamente em fornalhas ou aproveitado para produção de carvão vegetal. O poder calorifico superior é de cerca de $5.447,38 \mathrm{kcal} / \mathrm{kg}$ (base seca).

\subsection{Carvão Ativado}

O CA tem maior gama de aplicações na área industrial sendo utilizado principalmente para tratamento de substanciais químicas indesejadas, pois possui um grande poder de adsorção (SOARES, 2002).

Este carvão pode ser empregado no tratamento de água para remoção de pesticidas e micro poluentes, inclusive melhorando algumas propriedades organolépticas como: cor, odor e sabor. Adicionalmente, pode ser utilizado no pré-tratamento da água utilizada nas indústrias de alimentos, bebidas, farmacêuticas e na osmose reversa removendo substâncias orgânicas (SOARES, 2002).

O uso do cloreto de zinco no carvão faz com que haja dilatação da estrutura molecular da celulose em consequência da quebra das ligações laterais da molécula criando a micro porosidade (FERNANDES, 2008)

A remoção de impurezas nas substancias usando CA ocorre devido ao processo de adsorção, as impurezas ficam retidas nas porosidades do carvão.

\section{METODOLOGIA}

As cascas de coco verde foram recolhidas de vendedores locais da cidade de Apucarana, Estado do Paraná, Brasil. Antes de serem submetidas ao processo de pirolise as cascas passaram pelo corte e secagem ao sol por 72 horas.

$\mathrm{O}$ processo de pirolise foi realizado utilizando latas de tintas reaproveitadas (lata de tinta de $18 \mathrm{~L}$ ) com furos de pequeno diâmetro que permitiam a entrada de pequena quantidade de ar atmosférico. A lata ficou aberta até a saída completa de fumaça branca (água e voláteis) e foi fechada até a completa pirólise das cascas de coco verde. A chama inicialmente induzida é apagada obstruindo a entrada de ar atmosférico (furos na base da lata de aço). Os carvões obtidos no final do processo anterior foram macerados e peneirados para posteriores testes de capacidade de adsorção. 


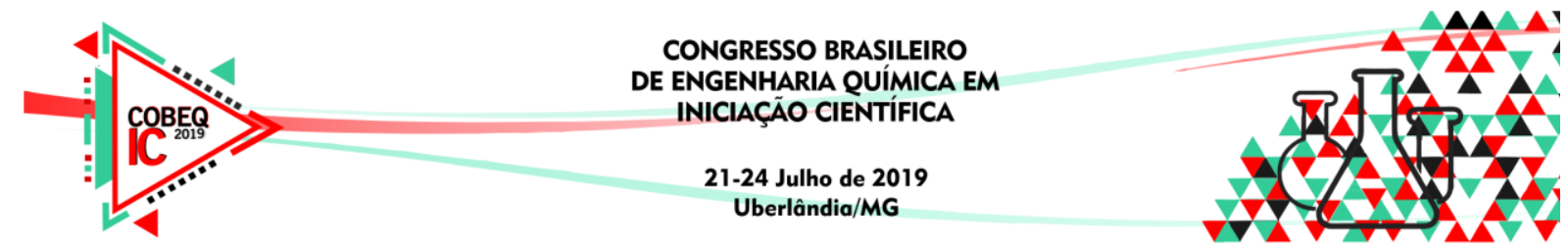

O material precursor foi divido em três lotes $(1,2,3)$ com massas iguais de $0.2500 \mathrm{~g}$ e cada lote foi impregnando com diferentes concentrações em base mássica de $\mathrm{ZnCl}_{2}, 50 \%$, $37,5 \%, 25 \%$ respectivamente. Posteriormente as triplicatas de cada lote foram deixadas por 15 horas na estufa a $80{ }^{\circ} \mathrm{C}$.

O material após seco foi colocado em cadinhos e levado ao forno elétrico onde ficou exposto a uma temperatura de $500{ }^{\circ} \mathrm{C}$ por 30 minutos. Posteriormente foi lavado com solução de $\mathrm{HCl} 0,10$ mol. $\mathrm{L}^{-1}$ e com abundância de agua quente e fria alternadamente, o procedimento de lavagem se encerrou quando a agua residual atingiu o $\mathrm{pH}$ igual a 7 . As triplicatas foram novamente colocadas na estufa para secagem e obtenção do carvão vegetal ativado de cascas de coco verde.

As analises de adsorção foram realizadas utilizando como amostra rejeitos de lavanderia de uma indústria da região norte do Paraná. Os testes foram realizados tanto com o carvão vegetal ativado neste trabalho quanto com o seu similar comercializado.

As amostras (rejeito da lavanderia) foram diluídas em uma solução de concentração 1:100 (solução A), na qual se adicionou $10,00 \mathrm{~mL}$ em tubos tipo falcon nos quais foram colocadas diferentes massas de carvão (deste trabalho e comercial). As massas adicionadas foram: $1,00 \mathrm{~g}(\mathrm{~B}), 0,50 \mathrm{~g}(\mathrm{C})$ e $0,25 \mathrm{~g}(\mathrm{D})$.

Os diferentes tubos falcon foram agitados e dispostos em centrifuga da marca Quimis, Q222T para o processo de separação. A centrifuga operou por 40 minutos a uma velocidade de $3200 \mathrm{rpm}$.

As análises de adsorção foram realizadas em espectrofotômetro dual bean, Agilent Technologies (Cary 60), acondicionadas em cubetas de vidro 10mm, em $550 \mathrm{~nm}$.

No que se refere às adsorções com o carvão ativado de cascas de coco verde a amostra (rejeito de lavanderia) foi diluída em uma solução 10:100. Foram colocados $10 \mathrm{~mL}$ desta solução em 3 lotes $(1 \mathrm{~F}, 2 \mathrm{~F}, 3 \mathrm{~F})$ de tubos falcon. Em cada lote foi aplicado aproximadamente $0,2500 \mathrm{~g}$ de cada lote de carvão ativado, lotes $1,2,3$.

Em seguida as triplicatas de cada lote foram agitadas e colocadas em repouso de 72 horas para decantação, após a sedimentação do carvão e a clarificação da amostra, o liquido foi analisado em espectrofotômetro dual bean, Agilent Technologies (Cary 60), acondicionadas em cubetas de vidro $10 \mathrm{~mm}$, em $550 \mathrm{~nm}$.

\section{RESULTADOS E DISCUSSÃO}

O resultado do perfil espectroscópico do rejeito de lavanderia testado é mostrado conforme Figura 1. 


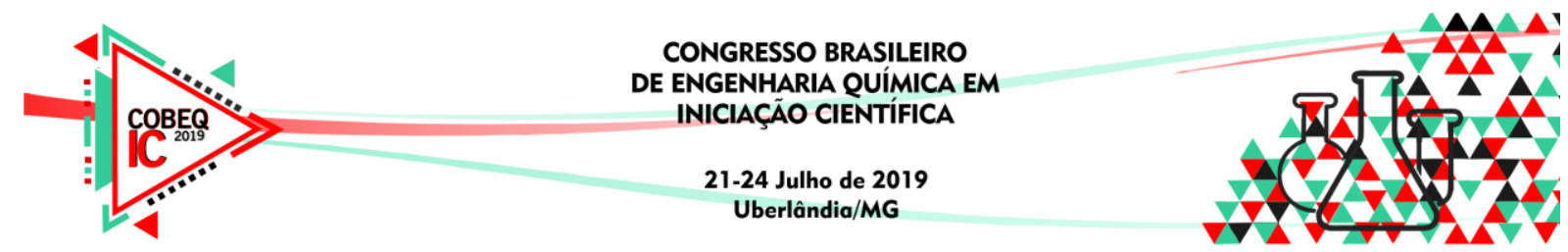

Figura 1 - perfil espectroscópico.

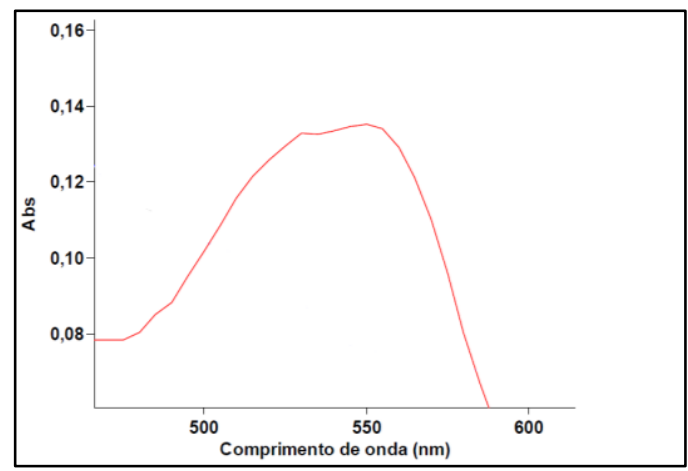

Com base na Figura 1, pode-se observar que no comprimento de onda $550 \mathrm{~nm}$ a amostra apresentou maior absorbância, sendo este empregado para as análises de quantificação espectroscópicas.

Com dados das análises prerrogativas optou-se por utilizar a massa de carvão igual 0,25 g em cada tubo falcon. Os resultados dos testes de adsorção podem ser observados conforme a Figura 2. Neste teste foram comparados o carvão ativado encontrado no mercado e o carvão de coco produzido neste trabalho, os resultados mostram que de todo o rejeito de lavanderia apenas $16 \%$ não foram adsorvidos pelo carvão de coco, enquanto que o carvão ativado comercial não conseguiu adsorver o montante de $26 \%$. Os resultados mostram superioridade na qualidade do carvão produzido neste trabalho mesmo este não sendo ativado quimicamente.

Os dados obtidos nos testes de adsorção podem ser aferidos conforme figura 2.

Figura 2 - resultados da análise em espectrofotômetro.

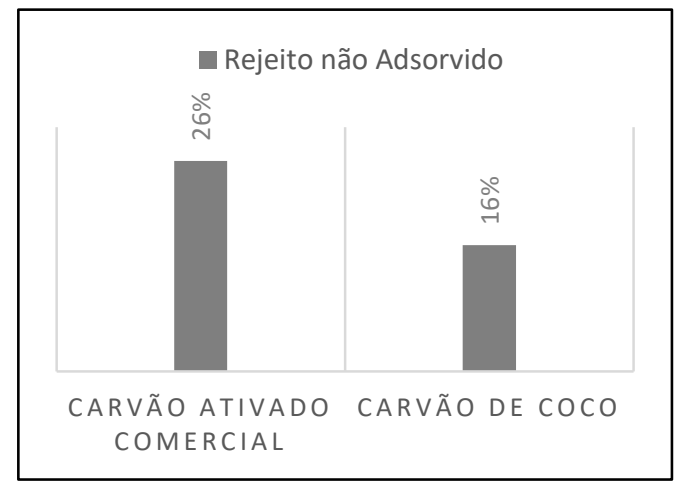

Os resultados da analises das amostras contendo carvão ativado podem ser visualizados conforme a Figura 3. Os dados indicam que quanto maior a quantidade utilizada de agente ativador no processo, maior será sua eficiência pois no melhor resultado obtido apenas 


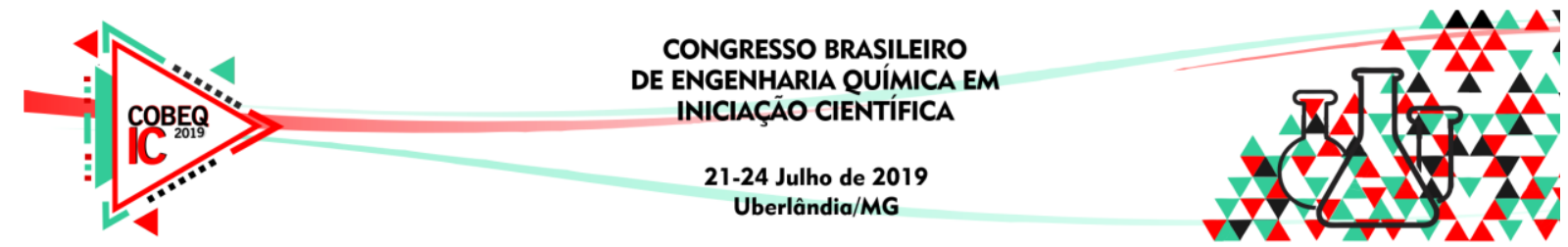

0,109\% do rejeito de lavanderia não foi adsorvido mostrando novamente uma alta eficiência comparado ao teste com carvão ativado encontrado no mercado.

Figura 3 - resultados da análise em espectrofotômetro do carvão de coco ativado.

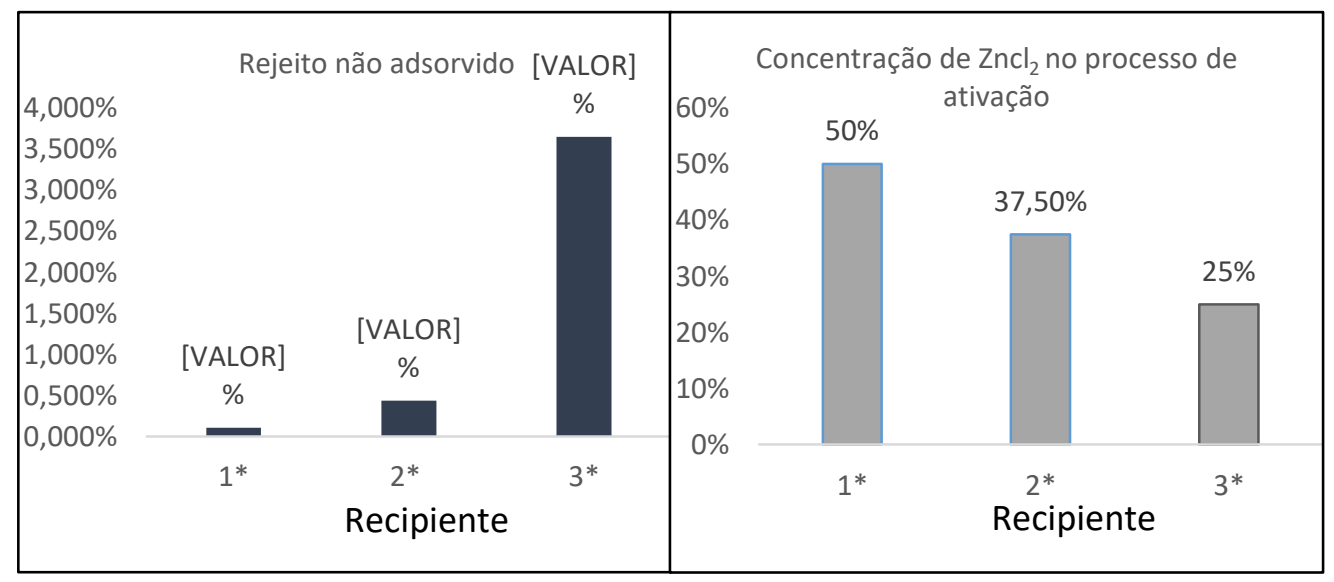

A Figura 4 compara a cubeta (lado esquerdo) que contém o material com rejeito da lavanderia com a cubeta (lado direito) na qual se aplicou o carvão ativado feito neste trabalho para adsorção das impurezas da amostra, tanto nos dados obtidos quanto na visualização após o tratamento fica evidente a eficiência do carvão no tratamento do resíduo.

Figura 4 - cubeta com rejeito a esquerda, cubeta após adição de carvão de coco ativado.

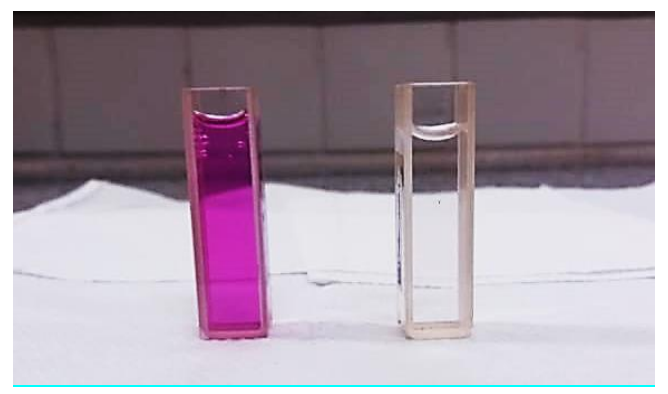

\section{CONCLUSÃO}

Nas condições das análises realizadas, os melhores resultados foram obtidos a partir do carvão de coco produzido neste trabalho. Tanto o carvão sem ativação quanto o carvão com ativação química apresentaram efeitos superiores em comparação ao carvão ativado encontrado no mercado (carvão comercial), os resultados das análises sugerem que dentre os 3 níveis de concentração do agente ativador, o melhor foi o contendo $50 \%$ de cloreto de zinco II. 


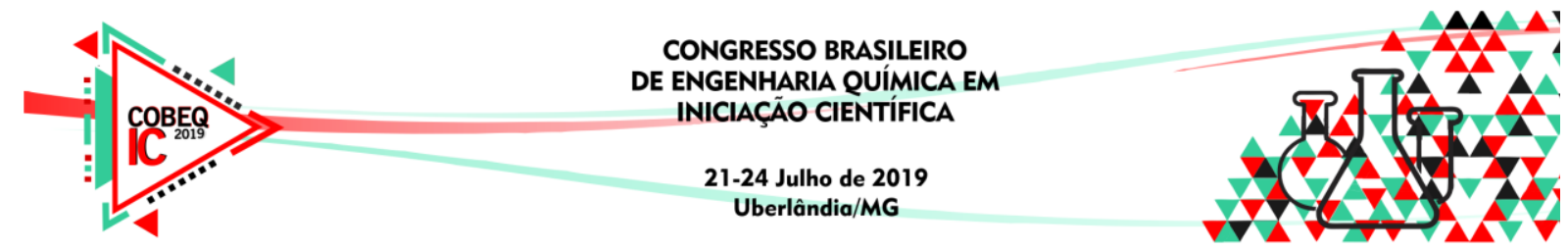

Os testes de adsorção com o carvão de casca de coco deste projeto se mostraram promissores para a purificação de rejeitos industriais de lavanderia.

\section{REFERÊNCIAS}

BAUDOUIN, L.; BATUGAL, P.A; OTHMAN, A.; SANTOS, G.A.; LABOUISSE, J.P. Manual on standardized research techniques in coconut breeding. 1996. Disponível em:

$<$ https://www.bioversityinternational.org/fileadmin/bioversity/publications/Web_versio n/108/ch02.htm\#Chapter\%201\%20BOTANY\%20OF\%20THE\%20COCONUT\%20PA

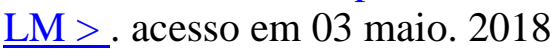

BBC BRASIL. Indústria do coco cresce, mas alto desperdício gera desafio tecnológico. Disponivel em: < https://economia.uol.com.br/noticias/bbc/2014/02/17/industria-dococo-cresce-mas-alto-desperdicio-gera-desafio-tecnologico.htm >

CARRIJO, O.A.; LIZ, R.S.; MAKISHIMA, N. Fibra da casca do coco verde como substrato agrícola. Horticultura Brasileira, Brasília, v. 20, n. 4, p. 533-535, Disponível em: <http://www.scielo.br/pdf/hb/v20n4/14486.pdf >.Acesso em 24, Mar, 2018.

PALMA, Ana.; OLIVEIRA, Miguel.; Fiocruz. Outros transmissores da dengue. 2014. Disponível

em: <http://www.invivo.fiocruz.br/cgi/cgilua.exe/sys/start.htm?infoid=1411\&sid=2

$>$ Acesso em 24, Mar, 2018.

ANDRADE. A. M.; PASSOS. P. R. A; MARQUES. L. G. C; OLIVERIRA. L. B; VIDAURRE.G.V;ROCHA.J.D.S. Revista arvore. Pirólise de resíduos do coco-da-baía (Cocos nucifera Linn) e análise do carvão vegetal. 2004.Disponivel em:< http://www.scielo.br/scielo.php?script=sci_arttext\&pid=S0100-67622004000500010 >. Acesso em 14, abril, 2018.

NOGUEIRA, L.A.H. et al. Dendroenergia: fundamentos e aplicações. Brasília, DF: ANEEL Agência Nacional de Energia Elétrica, 2000.

FERNANDES, F. L.; Tese de doutorado. Carvão De Endocarpo De Coco Da Baía Ativado Quimicamente Com Zncl2 E Fisicamente Com Vapor D'água: Produção, Caracterização, Modificações Químicas E Aplicação Na Adsorção De Íon Cloreto. 2008 Disponivel

em: <https://www.google.com.br/search?q=fabiana+lopes+fernandes+tese+de+doutorado\& oq=fabiana+lopes+fernandes+tese+de+doutorado\&aqs=chrome..69i57.7645j0j7\&sourc eid=chrome\&ie=UTF-8\# > . Acesso em 15, abril, 2018

SOARES, A. G..; Tese de doutorado. Adsorção de gases em carvão ativado de celulignina. 2002. 


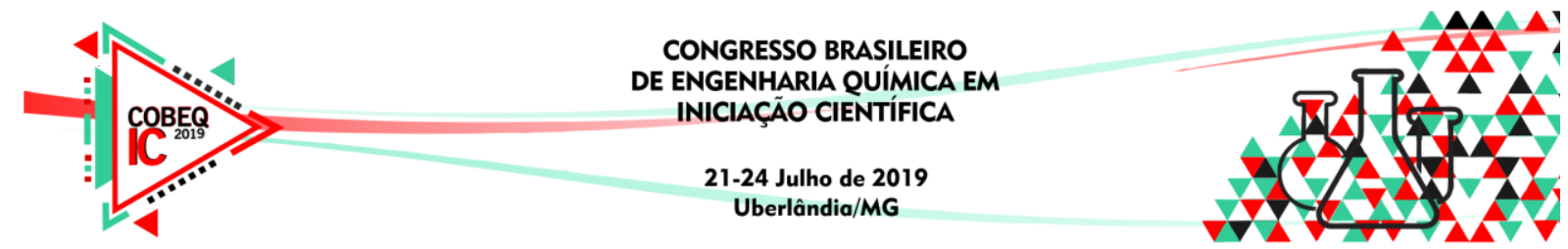

<http://repositorio.unicamp.br/bitstream/REPOSIP/277548/1/Soares_AlvaroGuedes_D. pdf $>$. Acesso em 20, abril, 2018 